\title{
VANISHING DIMENSIONS AND PLANAR EVENTS AT THE LHC
}

\author{
Greg Landsberg ${ }^{* \dagger}$ \\ Brown University, Department of Physics, 182 Hope St., Providence, RI 02912, USA \\ E-mail: landsberg@hep.brown.edu
}

\begin{abstract}
We present a paradigm in which the effective dimensionality of space depends on the length scale we are probing. At short scales the space is lower dimensional; at the intermediate scales the space is three-dimensional; and at large scales, the space is effectively higher dimensional. This setup allows for some fundamental problems in cosmology, gravity, and particle physics to be approached from a new perspective and results in striking collider phenomenology. It may also explain elongated jets observed in cosmic-ray data.
\end{abstract}

35th International Conference of High Energy Physics - ICHEP2010,

July 22-28, 2010

Paris France

\footnotetext{
* Speaker.

$\dagger$ This conference proceeding is based entirely on a recent work [1] done in collaboration with Luis Anchordoqui, De Chang Dai, Malcolm Fairbairn, and Dejan Stojkovic.
} 
Despite the fantastic success of the standard model of particle physics (SM) and the standard model of cosmology, various fundamental problems have accumulated that need attention. Many of these problems stem from the ultraviolet (short distance) and infrared (large distance) divergencies. There is a general consensus that we understand our Universe (with some exceptions) on scales approximately between $10^{-18} \mathrm{~m}$ and $10^{24} \mathrm{~m}$. It is becoming increasingly clear that straightforward extensions of existing theories either do not cure everything or bring in more problems. Some radically new ideas are needed to explain the Universe beyond these scales. We propose a novel approach introducing the concept of evolving dimensionality: the effective dimensionality of space depends on the energy scale we are probing and as the length scale increases new dimensions open up. At short scales the space is lower dimensional, at intermediate scales the space is $3 \mathrm{D}$, and at large scales the space is effectively higher dimensional. This set-up allows for some fundamental problems in particle physics, gravity and cosmology to be addressed from a new perspective.

One of the most acute problems connected with ultraviolet divergences concerns radiative corrections to the mass appearing in the Higgs potential, $V=-\mu^{2} \Phi \Phi^{\dagger}+\lambda\left(\Phi^{\dagger} \Phi\right)^{2}$. The one-loop corrections to the Higgs mass from the top, $W$, and Higgs self-coupling diagrams grow quadratically with the ultraviolet cutoff of the theory $\Lambda: \Delta \mu^{2}=\frac{3}{16 \pi^{2} v^{2}}\left(2 m_{W}^{2}+m_{Z}^{2}+m_{H}^{2}-4 m_{t}^{2}\right) \Lambda^{2}$ [2], where $m_{W}, m_{Z}, m_{H}$, and $m_{t}$ are the masses of the $W$ and $Z$ bosons, Higgs boson, and top quark, respectively, and $v$ is the vev of the Higgs field. Unless the Higgs mass is fine-tuned to an accuracy $\mathscr{O}\left(10^{-32}\right)$, these corrections result in a dangerous contribution to the Higgs vacuum expectation value, which destabilizes the electroweak symmetry breaking scale. The SM works amazingly well by fixing $\Lambda$ at the electroweak scale. It is generally assumed that this indicates the existence of new physics beyond the SM at energies above $\Lambda$. The alternative approach we exercise here is to keep the the structure of the SM and change the dimensionality of the background on which the SM lives. A straightforward calculation shows that in $2 \mathrm{D}$ all of the terms in the above expression are only linearly divergent, while in 1D all of these terms are only logarithmically divergent, thus alleviating the fine-tuning problem. Therefore, lowering the dimensionality of space-time universally cures ultraviolet divergences in practically all of the field theories.

The most elusive concept in modern physics - the consistent quantization of gravity - is much more within the reach in lower dimensions. Gravity in $(3+1)$ space-time is complicated, nonlinear, and perturbatively non-renormalizable theory. However, if the fundamental short-scale physics is lower dimensional, there is no need to quantize $(3+1) \mathrm{D}$ gravity at short distances and we should quantize $(2+1)$ and $(1+1) \mathrm{D}$ gravity instead. In $(2+1) \mathrm{D}$ the curvature tensor $R_{\mu v \rho \sigma}=\varepsilon_{\mu v \alpha} \varepsilon_{\rho \sigma \beta}\left(R^{\alpha \beta}+\frac{1}{2} g^{\alpha \beta} R\right)$, where $R^{\alpha \beta}$ is a Ricci tensor and $R$ is a Ricci scalar. Since the Weyl tensor is not present, any solution of the vacuum Einstein's equations is locally flat. Thus, $(2+1) \mathrm{D}$ space-time has no local gravitational degrees of freedom, i.e. no gravitational waves in classical theory and no gravitons in quantum theory. The number of degrees of freedom in such a theory is finite, quantum field theory reduces to quantum mechanics and the problem of nonrenormalizability disappears [3]. $(1+1) \mathrm{D}$ gravity is even more simple - the gravitational coupling is dimensionless and the action is a topological invariant that gives no dynamics to the $2 \mathrm{D}$ metric. Interestingly, there is an asymptotically safe theory of pure gravity in $(2+\varepsilon)$ space-time dimensions $(\varepsilon \ll 1)$; asymptotic safety can also be preserved in the presence of matter fields [5]. There is also a connection with the causal dynamical triangulation approach that yields the similar dynamical fractal structure of space-time [4]. However, the layered space-time structure we 
propose is not fractal, but has the properties of dimensions on the lattice.

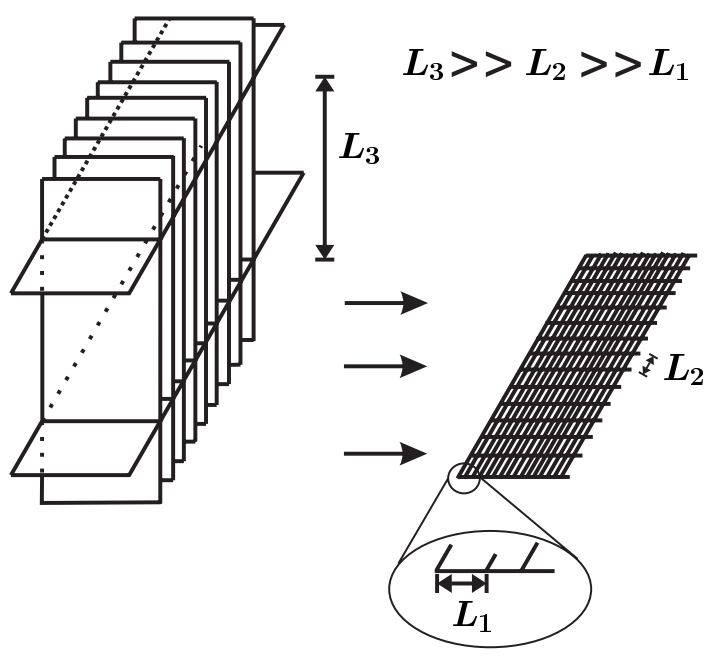

Figure 1: Ordered lattice. The fundamental quantization scale of space is indicated by $L_{1}$. Space structure is 1D on scales much shorter than $L_{2}=\Lambda_{2}^{-1}$, while it appears effectively 2D on scales much larger than $L_{2}$ but much shorter than $L_{3}=\Lambda_{3}^{-1}$. At scales much larger than $L_{3}$, the structure appears effectively 3D. Following this hierarchy, at even larger scales, say $L_{4}$, yet another dimension opens up and the structure appears $4 \mathrm{D}$ (not shown in the picture). From Ref. [1].

Let us assume space-time has an ordered lattice structure, see Fig. 1, which becomes anisotropic at very small distances. The proposed set up is analogous to that of dimensional crossover in layered strongly correlated metals: these materials have an insulating character in the direction perpendicular to the layers at high temperatures but become metal-like at low temperatures, whereas transport within the layers remains metallic over the whole temperature range. One could think about the changing dimensionality of space in this picture as evolving dimensionality of a folded tapestry. If one looks at it through a microscope, all one would see is an individual 1D thread; zooming out would reveal the 2D structure of the canvas; looking from further afar would show that the tapestry is folded into a complex 3D object. This analogy shows that the very "tapestry of space" can be fundamentally made of a very long $1 \mathrm{D}$ string.

For $\Lambda_{3}<\sqrt{s}<\Lambda_{2}$ the Universe is $2 \mathrm{D}$ and gravity, like any other force, is bound to $2 \mathrm{D}$. (Note that this is not the case of bulk+branes, as here there is no bulk.) The world is truly 2D in a sense that the only third dimension is the thickness of the brane $\sim \Lambda_{3}^{-1}$ (i.e., the thickness of the spacing between lattice sheets). The Minkowski space-time metric shrinks to $(1,-1,-1)$. From Gauss's law, the gravitational potential becomes $\phi(r)=2 G_{2} M \ln r$, where $G_{2}$ is the two dimensional gravitational constant. Gravity is still attractive, but is stronger as the force drops as $1 / r$, not $1 / r^{2}$. The Universe, however, is very hot $\left(T>\Lambda_{3} \gtrsim 1 \mathrm{TeV}\right)$ and so the earliest observationally verified landmarks - nucleosynthesis and cosmic microwave background — stay unaffected.

The local random orientation of the $2 \mathrm{D}$ substructure activates non-systematic violations of Lorentz symmetry in the low energy effective theory, i.e., for de Broglie wavelengths $\lambda>L_{3}$ (in the preferred frame of lattice coordinates) [6]. Hence, the observed light from distance sources is continually subject to fluctuations of the layered structure, which introduce an uncertainty $\delta \lambda$ in 
the determination of the photon wavelength [7]. The strongest constraints come from the phase coherence of light from distance sources, e.g. PKS1413+135, a galaxy at a distance of $1.2 \mathrm{Gpc}$ that shows Airy rings at a wavelength of $1.6 \mu \mathrm{m}$, is a typical probe. For $\Lambda_{3} \sim 1 \mathrm{TeV}$, the allowed region of the parameter space, encompasses the particularly interesting model of $\delta \lambda \sim L_{3}$. Similar, but weaker constraints can be also derived from the limits on time dispersion of a signal from a distant astrophysical source, e.g., from high-precision timing observations of the pulsar B1937+21 [8].

If in the preferred frame a de Broglie wavelength of a particle becomes significantly shorter than $L_{3}$, such a particle will propagate locally in $2 \mathrm{D}$, rather than $3 \mathrm{D}$. Note that this does not affect the straightness of propagation of high-energy gamma rays from the source to the observer, as the overall momentum of the particle is preserved as it propagates through the spatial lattice. If the lattice is rigid enough, i.e., the tension of the branes that form it is significantly higher than particle energy, the particle will scatter coherently at brane junctions and move along a jagged line preserving its original direction. This is similar to a photon propagating straight through a crystal lattice despite being scattered elastically off the individual atoms via phonon exchange. As long as the energy of the photon is small enough so that the scattering is elastic, the propagation of the electromagnetic wave through the crystal preserves the group velocity of the photon and its direction on the scales significantly larger than the lattice spacing. That allows us to elude all the astrophysical constraints from $\mathrm{TeV}$ gamma rays.

For $\sqrt{s}>\Lambda_{2}$, when space becomes $1 \mathrm{D}$, Minkowski metric is simply $(1,-1)$ and space and time in a sense become equivalent to each other. Interestingly, if $C P$ was violated maximally in the Big Bang, then $T$ is also violated maximally (assuming the $C P T$ theorem still applies) and the fact that the time has a defined direction, while space does not, may simply come from that maximum violation (just as neutrinos are always left-handed due to the maximum violation of parity in weak interactions).

For distances $>L_{4}$, space becomes $4 \mathrm{D}$, which would result in certain consequences for cosmology: indeed, the cosmological constant seen by a $(3+1) D$ observer can be viewed as a Casimir force due to the presence of another distant fold of the lattice. Interestingly enough, if $L_{4} \sim 10^{26} \mathrm{~m}$, i.e. comparable to the current horizon size, the right order of magnitude value for the cosmological constant is obtained.

The lower dimensional crossover has possible implications for LHC physics if $\Lambda_{3} \sim 1 \mathrm{TeV}$. Consider a $2 \rightarrow 2$ scattering in our brane-lattice model. If $Q^{2}$ of the scattering, i.e., the degree of virtuality of the mediator (propagator) in the corresponding Feynman diagram becomes comparable to $\Lambda_{3}^{2}$, the mediating particle moves in 2D. It's easy to see from dimensional analysis that partonlevel cross sections for 2D-scattering changes compared to that in 3D: for instance, the DrellYan cross section will drop not as $1 / E^{2}$, but as $1 / E^{3}$. The fact that this phenomenon has not been observed in the previous low-energy measurements, e.g., at LEP and the Tevatron, can be interpreted as the bounds on the sharpness of the $3 \mathrm{D} \rightarrow 2 \mathrm{D}$ crossover. Note that the fact that the propagator is bound to $2 \mathrm{D}$, while the incoming particles move in $3 \mathrm{D}$ on the distances much greater than $L_{2}$ does not result in $T$ and $C P T$-violation, as the outgoing particles also propagate in 3D over long distances, due to the lattice back-reaction, which absorbs the momentum of the incoming particles in the direction perpendicular to the local 2D fold and then reemit it by giving the outgoing particles equal boost in the same direction.

Let's move on to the $2 \rightarrow 4$ scattering, which involves several virtual particles. If $Q^{2}$ in each of 
the propagators is comparable with $\Lambda_{3}^{2}$, the spatial separation between the incoming and outgoing particles at the time of the interaction is comparable to the size of the lattice $L_{3}$. Thus, all the virtual particles (propagators) must move in the same 2D space transverse to the third dimension of the lattice, $L_{3}$. This results in the outgoing four partons to be in the same plane in the c.o.m. frame of the collision, thus drastically different from the 3D scattering, where four outgoing partons are in general acoplanar. As discussed above, the entire c.o.m. frame is boosted to conserve the longitudinal momentum of the incoming partons in the direction of the beam, but that does not affect the initial planar configuration, per the argument of photon propagation through the lattice. Thus, we expect, e.g., multijet events with four or more jets at very high transverse momentum to become more and more planar as the characteristic $Q^{2}$ approaches $\Lambda_{3}^{2} \sim 1 \mathrm{TeV}^{2}$. This phenomenon is studied in more detail in our follow-up work [10].

Finally, if the lattice structure is similar over large distances (which is is generally not the case, as the lattice surfaces forming it may be folded and twisted in a non-trivial way), i.e., over the distances comparable to $1 / \Lambda_{\mathrm{QCD}}$, individual jets at very high energy may become elliptic in shape. This is due to the nature of the parton shower, which is generally ordered in $Q^{2}$; thus one expects the largest $Q^{2}$ 's to happen at the beginning of the shower evolution. If several successive shower splittings have $Q^{2} \sim \Lambda_{3}^{2}$ and the lattice orientation is preserved over the distance scale of the shower development, just like the multijet events become planar, the core of the jet will become planar as well. After the soft part of the parton shower is finished, the resulting jets will be elliptic rather than round in shape. We believe that looking at the individual jet ellipticity as a function of jet energy may become an interesting experimental probe of models with vanishing dimensions. In fact, such elliptic jets may have been already observed by the Pamir Collaboration in showers induced by high-energy cosmic rays (the effect know as "alignment") [9], which can not be explained by conventional physics.

This work is partially supported by the US Department of Energy, under Grant No. DE-FG0291ER40688. The author is indebted to his collaborators on this project and to the organizers of the 35th ICHEP Conference for an exciting meeting and excellent organization.

\section{References}

[1] L. Anchordoqui et al., e-Print: arXiv:1003.5914 [hep-ph].

[2] M.J.G. Veltman, Acta Phys. Polon. B 12, 437 (1981).

[3] S. Carlip, J. Korean Phys. Soc. 28, S447 (1995).

[4] J. Ambjorn, J. Jurkiewicz and R. Loll, Phys. Rev. Lett. 85, 924 (2000); ibid 95, 171301 (2005).

[5] S. Weinberg, in General Relativity, (eds. S.W. Hawking and W. Israel, Cambridge University Press, 1979) p.790.

[6] D. Mattingly, Living Rev. Rel. 8, 5 (2005).

[7] Y.J. Ng, H. van Dam and W.A. Christiansen, Astrophys. J. 591, L87 (2003).

[8] V.M. Kaspi, J.H. Taylor and M.F. Ryba, Astrophys. J. 428, 713 (1994).

[9] See, e.g., A. De Roeck et al., in Proc. of the 13th International Conference on Elastic and Diffractive Scattering, Blois, 2009, arXiv:1002.3527 and references therein.

[10] L. Anchordoqui et al., e-Print: arXiv:1012.1870 [hep-ph]. 